\title{
P 012 FRAILTY NARRATIVES TOWARDS THE END OF LIFE
}

Anna Lloyd, Marilyn Kendall, Scott Murray. The University of Edinburgh, Edinburgh, United Kingdom

\subsection{6/bmjspcare-2014-000654.53}

Background Palliative care services have widened beyond cancer in recent years yet frail older adults rarely receive such services. There is a need to understand the multidimensional end of life experiences of this group in order to assess how or if a palliative approach could be beneficial.

Aims To describe the changing multidimensional experiences of frail older adults towards the end of life. 
Methods 76 in-depth qualitative interviews were carried out with 13 core participants and their key formal and informal carers repeatedly for a period lasting up to 18 months. Core participants were cognitively intact, community dwelling older adults considered to be moderately or severely frail using a clinical frailty scale. The interviews were participant led, audiorecorded and fully transcribed. The 'voice centred relational' narrative method, incorporating analyses of multidimensional experience, was used to compile case studies.

Results Narratives of frail older people approaching the end of life illustrate patterns of multidimensional experience that differ from the end of life trajectories of other groups. All participants experienced physical decline however three possible patterns of psychological, social and existential experience emerged. These were: stable; regressive and tragic; or regressive; according to the capacity to hold on to core values, and maintain a sense of self and of belonging in the world. Areas of greatest unmet need focused around psychological, social and existential dimensions while provision of care mainly focused on physical need.

Conclusion The current palliative care model may not be appropriate to meet the needs of frail older people. Considering the core values and sense of self that are involved in adjusting to and coping with losses experienced at this stage may help to highlight where best to alleviate psychological, social and existential distress as frail older people reach the end of life. 